\title{
Effectiveness of GP access to magnetic resonance imaging of the knee: a randomised trial
}

\author{
DAMASK (Direct Access to Magnetic Resonance Imaging: \\ Assessment for Suspect Knees) Trial Team
}

\begin{abstract}
\section{Background}

GPs commonly see patients with knee problems. Magnetic resonance imaging (MRI) of the knee is an accurate diagnostic test for meniscus and ligament injuries of the knee, but there is uncertainty about the appropriate use of MRI and when it should enter the diagnostic pathway for patients with these problems.
\end{abstract} Aim

To assess the effectiveness of GP referral to early MR and a provisional orthopaedic appointment, compared with referral to an orthopaedic specialist without prior MRI for patients with continuing knee problems.

Design of study

Pragmatic multicentre randomised trial with two parallel groups.

Setting

A total of 553 patients consulting their GP about a continuing knee problem were recruited from 163 general practices at 11 sites across the UK.

\section{Method}

Patients were randomised to MRI within 12 weeks of GP referral including a provisional orthopaedic appointment, or orthopaedic appointment without prior $\mathrm{MRI}$ within a maximum of 9 months from GP referral. The primary outcome measures were the Short Form 36-item (SF-36) physical functioning scale and the Knee Quality of Life 26-item Questionnaire (KQoL-26) at 6,12 , and 24 months.

Results

Patients randomised to MRI improved mean SF-36 physical functioning scores by 2.81 ( $95 \%$ confidence interval $[\mathrm{Cl}]=-0.26$ to 5.89 ) more than those referred to orthopaedics $(P=0.072)$. Patients randomised to MRI improved mean KQoL-26 physical functioning scores by $3.65(95 \% \mathrm{Cl}=1.03$ to 6.28$)$ more than controls $(P=0.007)$. There were no other significant differences.

\section{Conclusion}

GP access to MRI yielded small, but statistically significant, benefits in patients' knee-related quality of life but non-significant improvements in physical functioning.

\section{Keywords}

family practice; knee injuries; magnetic resonance imaging; randomised controlled trial.

\section{INTRODUCTION}

Each year in the UK, 15\% of patients who consult GPs do so for musculoskeletal disorders. Knee problems are common: patients aged >55 years often suffer from osteoarthritis, while at younger ages injury to the menisci or ligaments - often described as 'internal derangement' - is more common. The annual consultation rate for internal derangement of the knee is 32 per 1000 patient years, similar to rheumatoid arthritis. ${ }^{1}$ Imaging of the knee is a common musculoskeletal application of magnetic resonance imaging (MRI). ${ }^{2}$ Despite evidence for the technical and diagnostic performance of MRI for patients with injuries to the menisci and cruciate ligaments, ${ }^{3,4}$ there is uncertainty about the appropriate use of MRI and when it should enter the diagnostic pathway for patients with suspected internal derangement of the knee.5,6

Systematic reviews have shown that MRI is sensitive and specific in detecting lesions of the cruciate ligaments and menisci. ${ }^{7-9} \mathrm{MRI}$ correctly classifies at least $85 \%$ of meniscal lesions and $90 \%$ of healthy menisci found at arthroscopy. ${ }^{8}$ This has led to suggestions that access to MRI could inform GPs, improve their diagnosis and management, and ultimately improve patient health. ${ }^{10}$

Negative MRI findings could allow GPs to reassure

DAMASK (Direct Access to Magnetic Resonance Imaging: Assessment for Suspect Knees) Trial Team, York Trials Unit, Department of Health Sciences, University of York, York.

Address for correspondence

Dr Stephen Brealey, York Trials Unit, Department of Health Sciences, Seebohm Rowntree Building, University of York, York, YO10 5DD. E-mail: sb143@york.ac.uk

Submitted: 14 March 2008; Editor's response: 5 May 2008; final acceptance: 15 July 2008.

(C)British Journal of General Practice.

This is the full-length article of an abridged version published in print. Cite this article as: Br J Gen Pract 2008; DOI: 10.3399/bjgp08X342651 
patients, treat them conservatively in primary care, avoid unnecessary orthopaedic referrals, reduce waiting times, and save costs, ${ }^{11}$ positive MRI findings could confirm GPs' clinical diagnoses and decisions to refer to orthopaedic specialists, who could decide whether arthroscopy was needed without the need for a second appointment. The radiologist's report could also help to prioritise outpatient appointments. ${ }^{12}$ In contrast, patients' health might benefit more from immediate referral by their GP to an orthopaedic specialist. $^{6}$ This would allow hospital specialists to use MRI more selectively, thereby reducing resources spent on the procedure. Imaging may also confuse the clinical picture if it detects asymptomatic abnormalities, possibly leading to unnecessary referrals and interventions. ${ }^{13}$ As not all GPs understand $\mathrm{MRI}$ findings that are reported by radiologists, this could result in false reassurance and delays in appropriate treatment. ${ }^{14}$

A survey of MRI availability reported that of 121 public-sector departments with MRI that responded, $74(61 \%)$ provided direct access to GPs for imaging of the knee. ${ }^{15}$ Primary care trusts will control more than $80 \%$ of the NHS budget by $2007 / 2008 .{ }^{16}$ In addition, the Department of Health has plans to reduce waiting times for diagnostic tests and to spend $£ 2400$ million on upgrading diagnostics over the next 5 years. ${ }^{17}$ Over 1 million MRI examinations are performed in England each year, and provision is expected to rise. ${ }^{18}$ There is a real danger that GP access to MRI will become standard policy without rigorous evaluation. However, timely access to a reliable diagnostic tool in primary care could result in better care and reduced costs.

This study evaluated direct access by GPs to MRI as a complex intervention. ${ }^{19}$ The primary objective was to contribute to decisions by the National Institute for Health and Clinical Excellence by evaluating whether GPs' use of MRI for patients with suspected internal derangement of the knee improves their general physical or knee-related health. Recognising that improved care is the route to improved outcomes, the study also assessed whether GPs' use of MRI affects their subsequent diagnosis and management plans, as presented in an earlier article. ${ }^{20}$ The accompanying article of cost-effectiveness analysis reports whether MRI reduces net costs to the NHS. ${ }^{21}$

\section{METHOD}

A pragmatic randomised trial was conducted in urban, mixed, and rural sites across North East Scotland, North Wales, and Yorkshire, covering a broad socioeconomic spectrum. In these geographical areas there is around 2 million people who are registered in over 600 general practices. Full details of the trial methods are presented elsewhere. ${ }^{22}$

\section{How this fits in}

Imaging of the knee is a common musculoskeletal application of magnetic resonance imaging (MRI). There is evidence that MRI of the knee is an accurate diagnostic test for menisci and ligament injuries but there is also uncertainty about the appropriate use of MRI and, in particular, when it should enter the diagnostic pathway for patients with continuing knee problems. In this study a similar number of patients attended orthopaedic appointments whether they had direct access to MRI or were referred to an orthopaedic specialist, so access to MRI did not affect GPs' decisions about the need to refer to a hospital specialist. GP access to MRI did not bring about a significant improvement in patients' physical functioning and resulted in only small, but statistically significant, benefits in patients' knee function.

\section{Interventions}

Direct access to MRI (experimental intervention). The aim at each hospital was to perform imaging within 12 weeks of GP referral using standard, commerciallyavailable magnetic resonance machines and imaging protocols at the discretion of the radiologist. GPS advised patients with normal MRI findings to undergo conservative treatment including quadriceps exercises, to take up a referral to physiotherapy depending on clinical history, and to return to normal activities. GPs were advised that when radiologists reported a serious abnormality (such as a tumour) on an MRI examination, the patient should be fasttracked as usual. Whatever the MRI findings, the provisional orthopaedic appointment that had been made at the time of randomisation could be kept or cancelled at the discretion of the GP.

Educational interventions to support the dissemination of clinical guidelines can improve GPs' knowledge of MRI use. ${ }^{23}$ As such, at the start of the trial a consultant radiologist, consultant orthopaedic surgeon, and trial coordinator delivered 2-hour educational seminars to GPs about MRI, clinical diagnosis, and conservative management. Practices were offered a $£ 50$ payment or continuing medical education points if a GP attended the seminar; attendance was not a condition for participating in the trial. The seminars were used to raise GP awareness of when MRI is useful in the imaging of the knee for meniscus and ligament injuries, and addressed the clinical assessment and management of these knee problems.

The routine attachment of educational messages to radiologists' reports has been found to avoid unnecessary referral by GPs. ${ }^{24}$ Therefore, an educational message was attached to radiologists' reports, reminding the referring GP that the decision to proceed to orthopaedic referral or to continue with conservative treatment in primary care depended on both the MRI and clinical findings. If the orthopaedic referral was considered unnecessary, the provisional 


\section{Box 1. Eligibility criteria.}

\section{Inclusion criteria}

Patients were eligible if:

- their ages were between 18 and 55 years inclusive;

- their GP was considering referral to an orthopaedic specialist for suspected internal derangement of the knee (for example, meniscal or ligamentous injuries).

\section{Exclusion criteria}

Patients were not eligible if:

- their GP judged that they needed urgent orthopaedic referral at the initial consultation (for example, gross ligamentous injury or sudden onset of effusion);

- they had suspected osteoarthritis, other non-traumatic arthropathy, or isolated patello-femoral joint pain;

- they had chronic instability of the knee due to history of major injury;

- they had a previous MRI examination within the same episode of care;

- they had previous surgical intervention (excluding diagnostic arthroscopy) on the same knee;

- they had contraindications to the use of MRI; for example, pacemaker, intracranial aneurysm clips, or orbital metallic foreign body.

orthopaedic appointment should be cancelled.

Referral to orthopaedic specialist in secondary care (the control intervention). The aim was to ensure that the orthopaedic appointment was within a maximum of 9 months from GP referral. Orthopaedic specialists could request an MRI examination for patients in the control arm if clinically indicated.

\section{Recruitment, randomisation, management, and follow-up}

Patients were recruited from general practices between November 2002 and October 2004. In each practice, GPs or practice nurses asked those patients who were eligible (Box 1) and consented to participation to complete a baseline questionnaire before being randomised to the local radiology department for MRI or referral as usual to an orthopaedic specialist.

The GP made the subsequent referrals for orthopaedic clinic and MRI as necessary. To avoid contaminating the evaluation by differences in waiting times between the two clinical policies, GPs made a concomitant provisional referral to orthopaedics when requesting $\mathrm{MRI}$ of the knee. As the trial was pragmatic in design to reflect the consequences of routine GP access to MRI, blinding of patients or professionals to treatment allocation was neither desirable nor possible.

The remote telephone randomisation service at York Trials Unit ensured immediate and unbiased allocation of those patients who consented to the study. The randomisation procedure was stratified by experimental site, distance from practice to hospital, and number of partners in the practice. Within strata, a block allocation sequence was used; permuted random blocks of size 2 or 4 were randomly selected to generate the allocation sequence.

Follow-up of patients was by postal questionnaire at 6,12 , and 24 months after randomisation. This was to ensure that the evaluation covered all events including arthroscopy. An evidence-based strategy was used to maximise response rates..$^{25}$ At 6 months this included a reminder by post after 2 and 4 weeks and by telephone after 6 weeks. At 12 and 24 months, this strategy was supplemented with a 2-week prenotification letter and a direct payment of $£ 5$ that was not conditional on the questionnaire being completed.

\section{Referral process}

Data were collected from hospitals on the number of MRIs, orthopaedic appointments, and arthroscopies attended in the two groups, as well as patient waiting times from randomisation to $\mathrm{MRI}$, orthopaedic consultation, and arthroscopy.

\section{Health outcomes}

Two generic measures were used: the Short Form 36item (SF-36) ${ }^{26}$ questionnaire, a popular health profile validated for use in the NHS to identify unexpected effects of interventions, and $\mathrm{EQ}-5 \mathrm{D},{ }^{27}$ a commonly used health status index for economic evaluation. Both measures are responsive to changes in the health of patients referred for MRI of the knee. ${ }^{28}$ In the absence of an appropriate, patient-assessed health instrument specific to the knee with satisfactory evidence for reliability, validity, and responsiveness, ${ }^{29}$ the researchers developed their own instrument: the Knee Quality of Life 26-item Questionnaire (KQoL-26). ${ }^{30}$

The primary outcome measures are the physical functioning scale of the SF-36 and KQoL-26. Patients were also asked to record the number of days they took off work or the number of days they were prevented from undertaking normal activities because of their knee problem.

\section{Sample size}

A similar group of patients followed up 6 months after referral for MRI of the knee had a mean of 64 and standard deviation (SD) of 25 on the physical functioning scale of the SF- $36 . .^{25}$ Hence, a trial that followed up 434 patients (217 direct access and 217 controls) would have $80 \%$ power using a $5 \%$ significance level to identify a standardised difference of 0.27 . This is equivalent to 6.75 points on the SF-36 physical functioning scale or a similar number of points on KQoL-26 to achieve a standardised difference of 0.27 which, it was judged in either measure, should be 
clinically important. As it was estimated that the research could achieve $85 \%$ response rates to postal questionnaires, the aim was to recruit 500 patients.

\section{Statistical analysis}

Data were collected at four times: at baseline, after 6 and 12 months, and between 22 and 24 months. The primary analysis was 'by intention to treat', in that all patients properly randomised were included in the analysis even if they did not receive their allocated intervention. To take account of repeated measures within patients, the analysis used the PROC mixed procedure in SAS (version 9). Patient effects were fitted as random effects, and all other variables were included as fixed effects. ${ }^{31}$ The primary outcomes were the SF-36 physical functioning score and the KQoL-26 at each follow-up. The model included baseline primary outcome scores, time since baseline, and centre. The analyses of the other seven subscales of the SF-36 were conducted in the same way. The fit of the resulting models to the data was checked.

\section{RESULTS}

\section{Patient flow and follow up}

Of 647 practices approached to take part in the trial, 285 (44\%) accepted the invitation. There was no significant difference in the number of GPs per practice between practices that did or did not take part $(P=0.24)$. However, there was a significant difference in distance: participating practices were

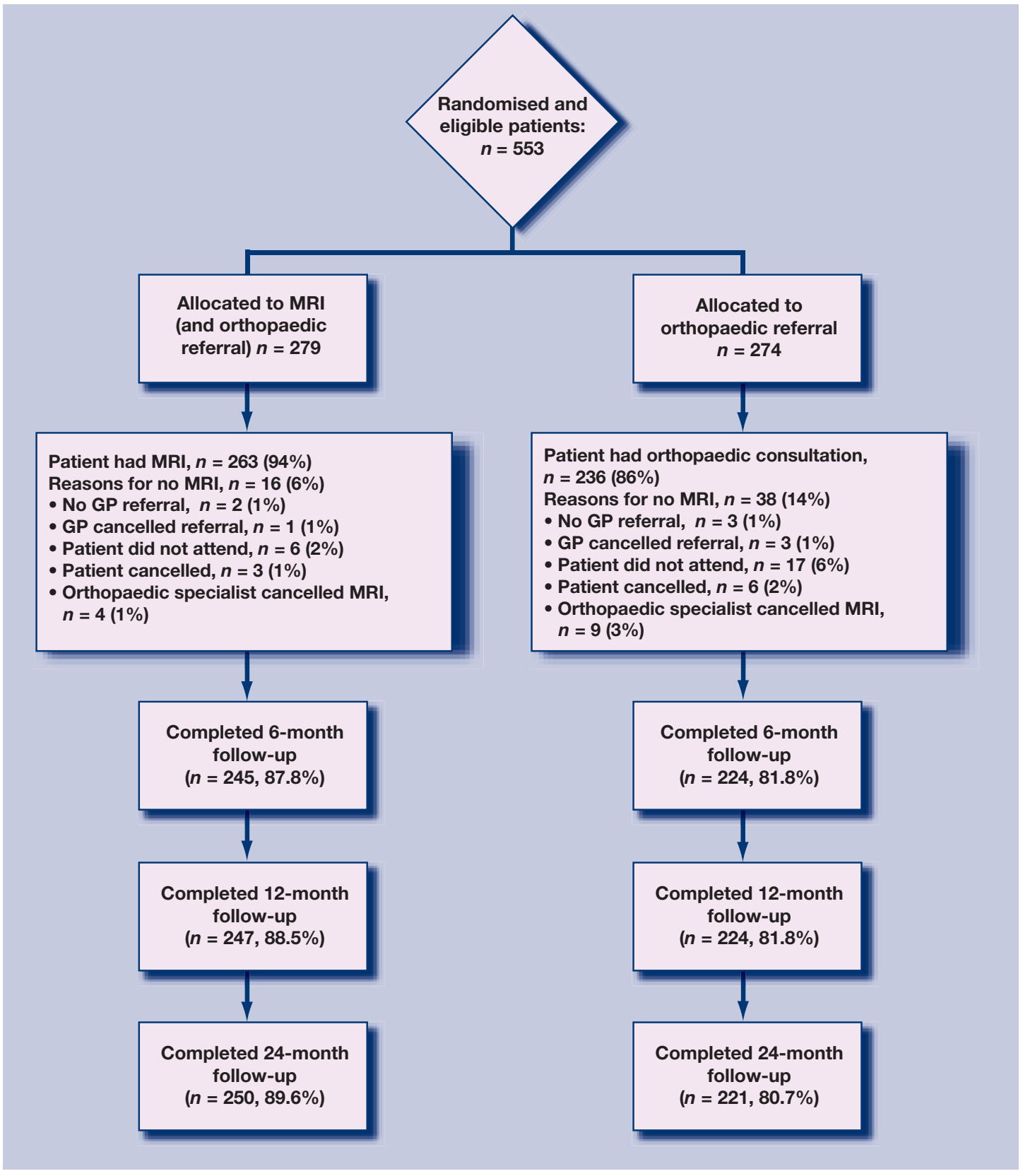

Figure 1. Flow of patients through trial. $M R I=$ magnetic resonance imaging. 
Table 1. Patient characteristics at baseline.

\begin{tabular}{lccc} 
Characteristics & $\begin{array}{c}\text { MRI group } \\
(n=279)\end{array}$ & $\begin{array}{c}\text { Orthopaedic group } \\
(n=274)\end{array}$ & $\begin{array}{c}\text { Total } \\
(n=553)\end{array}$ \\
\hline Sex: male, $n(\%)$ & $185 / 279(66.3)$ & $166 / 274(60.6)$ & $351 / 553(63.5)$ \\
\hline Ethnicity: white, $n(\%)$ & $269 / 272(98.9)$ & $266 / 270(98.5)$ & $535 / 542(98.7)$ \\
\hline Employed: yes, $n(\%)$ & $231 / 256(90.2)$ & $221 / 253(87.4)$ & $452 / 509(88.8)$ \\
\hline
\end{tabular}

Age, years

Mean (SD)

279

274

553

Diagnostic category ${ }^{\mathrm{a}}$

$40.2(10.1)$

$39.2(10.5)$

$39.7(10.3)$

Meniscal injury, $n(\%)$

Ligamentous injury, $n$ (\%)

Other diagnosis, $n(\%)$

$224(80.3)$

$87(31.2)$

$5(1.8)$

KQoL-26 (0-100, 100 = best health)

Physical functioning

Mean (SD)

Activity limitations

$n$

Mean (SD)

Emotional functioning

$n$

276

59.6 (19.1)

210 (76.6)

$82(29.9)$

$434(78.5)$ 9 (3.3)

$169(30.6)$

$14(2.5)$

Mean (SD)
SF-36 $(0-100,100=\text { best health })^{b}$
Physical functioning

Mean (SD)

Social functioning

Mean (SD)

Role physical

$n$

Mean (SD)

Role mental functioning

$n$

Mean (SD)

Mental health

$n$

Mean (SD)

Vitality

$n$

Mean (SD)

Pain

$n$

Mean (SD)

General health

$n$

276

$52.1(25.6)$

274

58.5 (18.9)

550

$59.1(19.0)$

273

$49.7(24.8)$

549

$50.9(25.2)$

277

$43.3(21.7)$

274

40.4 (22.7)

551

41.9 (22.2)

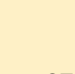

275
$55.4(23.7)$

274

$54.5(23.9)$

549

$54.9(23.8)$

273

274

$66.5(26.3)$

$54.46(23.9)$

547

$64.94(26.3)$

$$
275
$$

$52.6(29.5)$

271

$63.33(26.3)$

546
$51.84(29.7)$

275

272

547

$75.61(28.5)$

74.20 (26.3)

$74.91(27.4)$

277

274

551

$68.82(18.2)$

$67.63(19.8)$

68.23 (19.0)

277

274

51.68 (19.7)

$48.69(20.4)$

551

$50.19(20.1)$

270

45.50 (23.6)

270

43.55 (22.1)

540

44.52 (22.9)

EQ-5D (0-1, 1 = best health)

$n$

275

$71.02(19.3)$

273

$69.36(19.7)$

548 70.19 (19.5)

Days off work in last 4 weeks due to knee problem

Median (interquartile range)

163

$1(0-6)$

272

548

$0.6(0.2)$

$0.6(0.3)$

Days prevented from doing normal activities in last 4 weeks

\begin{tabular}{lccc}
$n$ & 230 & 211 & 441 \\
Median (interquartile range) & $7.5(2-15.3)$ & $8(2-20)$ & $8(2-16)$ \\
\hline
\end{tabular}

${ }^{a}$ Other diagnosis at trial entry: loose body, patella femoral problem, osteoarthritis, Baker's cyst, patellar ligament injury, worn meniscus. ${ }^{\mathrm{b}}$ The SF-36 physical and mental health summary scales use norm-based scoring; $50(S D=10)$ is the general US population mean. $M R I=$ magnetic resonance imaging. KQoL-26 $=$ Knee Quality of Life 26-item Questionnaire. SF-36 = Short Form 36-item health survey questionnaire. on average $2.3 \mathrm{~km}$ farther from a hospital $(P=0.035)$ compared to those practices that did not participate. From November 2002 to October 2004, 553 patients who were eligible and gave consent were recruited from 163 general practices; practices recruited between one and 23 patients, with 58 (36\%) recruiting four or more.

At 6 months, 469 (85\%) patients returned questionnaires; and at 12 and 24 months, 471 (85\%) patients returned questionnaires. Figure 1 shows patients' progress through the trial; most received their allocated intervention.

\section{Baseline data}

Table 1 shows the baseline characteristics of patients at randomisation. The mean (standard deviation [SD]) age of patients at randomisation was 39.7 (10.3) years and $36 \%$ were female. The mean (SD) SF-36 physical functioning score at randomisation was 54.9 (23.8). The two randomised groups had similar characteristics.

\section{Referral process}

In the MRI group, 230 patients (82\%) had an orthopaedic consultation compared with 236 patients $(86 \%)$ in the orthopaedic group, which was a difference of $3.7 \%(95 \%$ confidence interval $[\mathrm{Cl}]=$ -2.4 to 9.8). In the MRI group, 230 patients (82.4\%) had an orthopaedic consultation compared with 236 patients $(86.1 \%)$ in the orthopaedic group, which was a difference of $3.7 \%(95 \% \mathrm{Cl}-2.4 \%$ to $9.8 \%)$. In the MRI group, 112 patients (40.1\%) had an arthroscopy compared with 77 patients $(28.1 \%)$ in the orthopaedic group, which was a difference of $12.0 \%$ (4.1\% to $19.7 \%)$. In the MRI group, 20 of 108 (18.5\%) patients had a diagnostic rather than a therapeutic arthroscopy compared with 30 of $76(39.5 \%)$ in the orthopaedic group - a difference of $21.0 \%(95 \% \mathrm{Cl}=7.7$ to 33.8$)$. The median (interquartile range) waiting time from randomisation to MRI was 41 days (21-71 days) in the MRI group, and 170 days (103-312 days) in the orthopaedic group; from randomisation to orthopaedic consultation was 93 days (60-183 days) in the MRI group and 79 days (54-168 days) in the orthopaedic group; and from randomisation to arthroscopy was 337 days (219-458 days) in the MRI group and 294 days (174-468 days) in the orthopaedic group.

\section{Patient outcome}

Table 2 shows that over 24 months, patients randomised to MRI had mean SF-36 physical functioning scores that were better by $2.81(95 \% \mathrm{Cl}=$ -0.26 to 5.89 ) than those directly referred to orthopaedics (not statistically significant, $P=0.072$ ). Patients randomised to MRI also had mean KQoL-26 physical functioning scores that were better by 3.65 
(1.03 to 6.28; statistically significant, $P=0.007$ ). For these two patient outcomes, Figures 2 and 3 show changes over time for the two groups. There were no other significant differences in outcomes.

It was also found at the end of the study that, over the previous 12 months, the median number of days patients took off work or were prevented from normal activities was zero in both groups.

\section{DISCUSSION}

\section{Summary of main findings}

A similar number of patients attended orthopaedic appointments in both groups, so access to MRI did not affect GPs' decisions about the need for a referral to a hospital specialist. In contrast, orthopaedic specialists performed significantly more arthroscopies in the MRI group.

Patients randomised to MRI reported no significant improvement over time in the SF-36 physical functioning scale compared with those randomised to orthopaedic referral. Additionally, although a statistically significant improvement in the physical functioning scale of the KQoL-26 was achieved, this was not clinically significant.

\section{Strengths and limitations of the study}

A randomised trial design ensured that two similar groups of patients were compared and provided a rigorous basis for statistical inference. This design is rarely applied to the assessment of diagnostic imaging because of perceived ethical concerns about randomising patients to a non-imaging policy. ${ }^{32}$ Previous research that assessed the effect of GP access to MRI of the knee used an observational design and did not adequately address patient outcomes and costs. ${ }^{10}$ By recruiting over 500 patients, the present trial had power to detect small differences. The pragmatic design allowed comparison of effectiveness and costs of the two policies under routine conditions.

Patients were recruited from 163 general practices across the UK (median number of GPs per practice = four; median distance from practice to hospital $=10$ $\mathrm{km})$, thereby contributing to external validity. It was not feasible, however, to collect data on patients who were eligible but declined to participate. As such, those patients who were included might not be wholly representative of the population with suspected knee derangement. Although response rates were high, there was evidence of attrition bias, with fewer patients responding in the orthopaedic group than in the MRI group. Analyses showed that characteristics that might predict non-completion of questionnaires included treatment group, age, and sex.

Time constraints meant that the follow-up period was shorter by 2 months for 28 patients. In the present

\begin{tabular}{|c|c|c|c|c|}
\hline Outcome measures & $\begin{array}{c}\text { MRI group, } \\
\text { mean (SE) }\end{array}$ & $\begin{array}{c}\text { Orthopaedic } \\
\text { group, } \\
\text { mean (SE) }\end{array}$ & $\begin{array}{c}\text { Difference } \\
(95 \% \mathrm{Cl}) \\
\end{array}$ & $P$-value \\
\hline \multicolumn{5}{|l|}{ Primary outcomes } \\
\hline SF-36 Physical functioning & $72.23(1.50)$ & $69.41(1.52)$ & $2.81(-0.26$ to 5.89$)$ & 0.072 \\
\hline KQoL-26 Physical functioning & $75.72(1.28)$ & $72.07(1.30)$ & 3.65 (1.03 to 6.28$)$ & 0.007 \\
\hline KQoL-26 Activity limitations & $76.74(1.60)$ & $74.62(1.63)$ & $2.13(-1.15$ to 5.41$)$ & ) 0.20 \\
\hline KQoL-26 Emotional functioning & $71.76(1.58)$ & $69.16(1.61)$ & $2.60(-0.63$ to 5.84$)$ & 0.11 \\
\hline \multicolumn{5}{|l|}{ Secondary outcomes } \\
\hline SF-36 Social functioning & $79.48(1.53)$ & $79.13(1.56)$ & $0.35(-2.81$ to 3.51$)$ & 0.83 \\
\hline SF-36 Role physical & $74.98(1.77)$ & $73.97(1.80)$ & $1.01(-2.62$ to 4.64$)$ & 0.59 \\
\hline SF-36 Role emotional & $84.82(1.53)$ & $83.00(1.55)$ & $1.82(-1.32$ to 4.97$)$ & 0.26 \\
\hline SF-36 Mental & $72.74(1.08)$ & $72.38(1.10)$ & $0.36(-1.87$ to 2.59$)$ & 0.75 \\
\hline SF-36 Vitality & $54.53(1.22)$ & $53.85(1.24)$ & $0.68(-1.84$ to 3.19$)$ & 0.60 \\
\hline SF-36 Pain & $66.85(1.54)$ & $65.63(1.56)$ & $1.23(-1.95$ to 4.41$)$ & 0.45 \\
\hline SF-36 General Health & $68.46(1.06)$ & $67.63(1.08)$ & $0.83(-1.36$ to 3.02$)$ & 0.46 \\
\hline
\end{tabular}

$M R I=$ magnetic resonance imaging. SE = standard error. SF-36 = Short Form 36-item health survey questionnaire. KQoL-26 = Knee Quality of Life 26-item Questionnaire.

trial, GPs made provisional orthopaedic appointments at the time of the initial referral for MRI. This was done so that patients in the MRI group would not be disadvantaged by waiting longer for orthopaedic appointments. Furthermore, this should help to ensure that the total waiting time from GP consultation to orthopaedic appointment was similar for both trial arms, thereby avoiding contaminating the evaluation by differences in waiting times between the two clinical policies. It was found, however, that although GPs had the option to cancel the orthopaedic appointment after receiving the MRI report, they rarely did so. This is consistent with the findings from a study assessing the influence of MRI of the knee on GPs' decisions; although MRI significantly increased GPs' confidence in their decisions, it did not lead to their significantly altering their diagnoses or treatment plans compared with direct referral to an orthopaedic specialist. ${ }^{20}$ It is likely that MRI confirmed what GPS already knew, which was the need for a referral to an orthopaedic specialist.

The findings about subsequent referral from an orthopaedic specialist for an arthroscopy reveal that 88 of $250(35 \%)$ patients in the MRI group had a therapeutic arthroscopy, compared with 46 of 221 $(21 \%)$ in the orthopaedic group. The increased therapeutic arthroscopies in the MRI group might explain the slightly improved outcomes.

Finally, UK waiting times for MRI and orthopaedic referrals have been reduced over recent years as a result of various government initiatives. If the benefit in this research is predominantly from early intervention in patients in the MRI arm of the trial, one must remember that waiting times for orthopaedic consultation have improved since the trial was performed. As such, the net gain in patient outcome from early MRI in the UK might be smaller than reported here. 


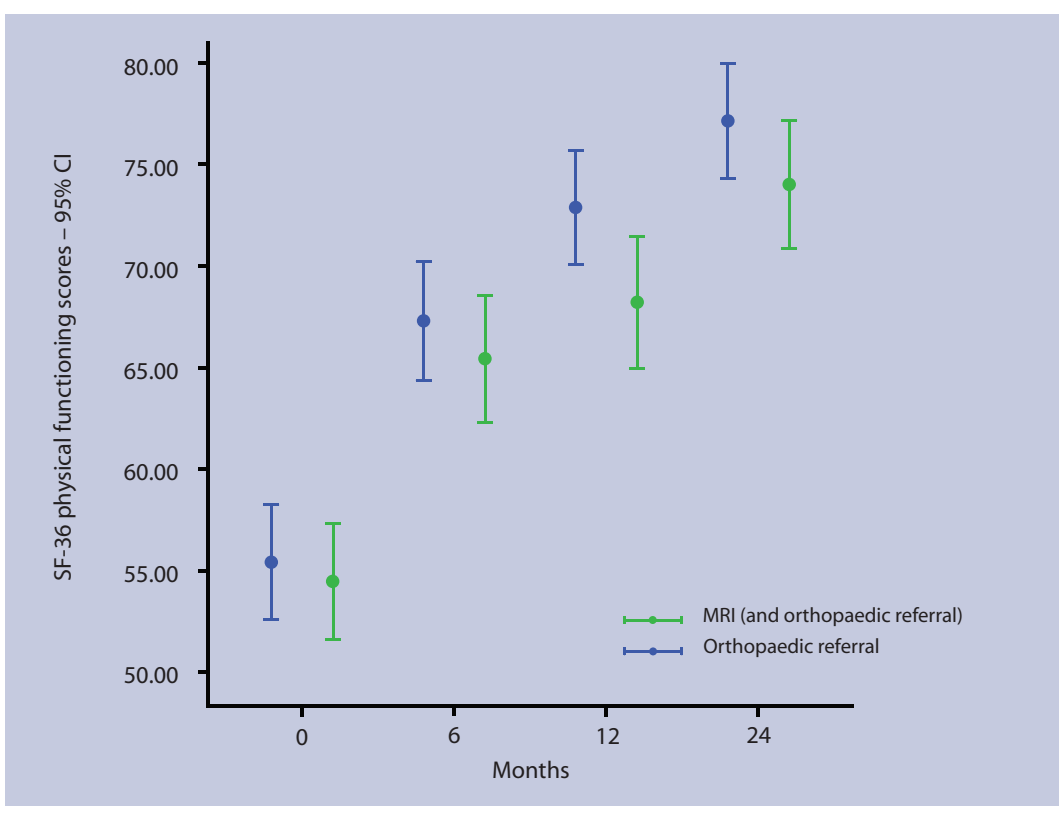

Figure 2. Mean Short Form 36-item (SF-36) physical functioning scores (with 95\% confidence intervals) over 24 months by group.

Figure 3. Mean Knee Quality of Life 26-item (KQoL-26) physical functioning scores (with 95\% confidence intervals) over 24 months by group.

\section{Comparison with existing literature}

Two small randomised trials were published about the effectiveness of MRI in the management of patients with suspected internal derangement of the knee. One included 209 patients recruited in secondary care with negative MRI results who were randomised for arthroscopy or conservative treatment, and concluded that MRI is accurate for the diagnosis of knee injuries. ${ }^{33}$ The other trial randomised 118 patients after referral by their GP or from the emergency department to a routine orthopaedic clinic, between MRI and arthroscopy; ${ }^{34}$ no significant differences in health outcomes were found between groups and there were similar mean costs. The present trial strengthens the evidence from these two

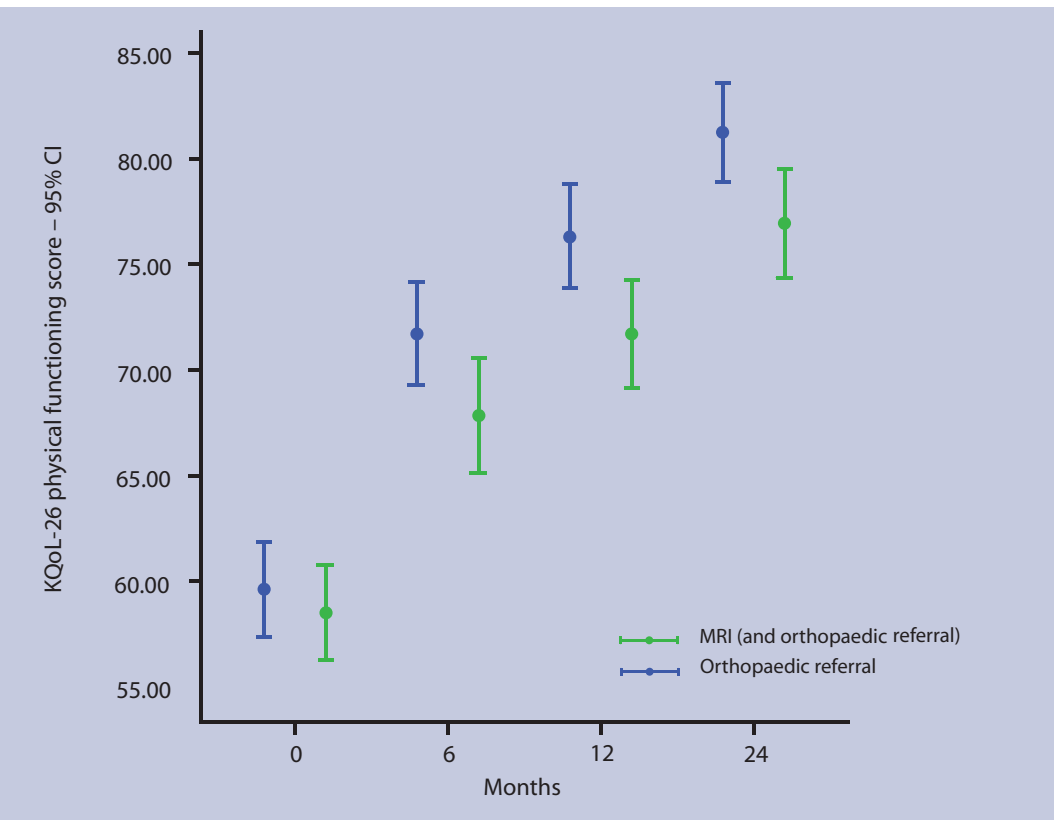

studies by focusing on recruitment from primary care with a substantially larger sample size.

\section{Meaning of the study}

The physical functioning scale of SF-36 comprises 10 items designed to measure limitations when performing daily activities, such as walking and climbing stairs, and scores those items between 0 and 100 , where 100 is best health. The study was designed to detect a difference of 6.75 points on this scale, equivalent to a standardised difference of 0.27 . GP access to MRI increased patients' scores on this scale by a net 2.81 points with a $95 \% \mathrm{Cl}$ of -0.26 to 5.89. This difference was not statistically significant $(P$ $=0.072$ ) and the Cls exclude the difference of 6.75 points adjudged clinically important.

The physical functioning scale of the KQoL-26 comprises 15 items designed to measure limitations when performing activities relating to the knee, such as walking, standing, kneeling, rather than general physical functioning. The KQoL-26 also scores between 0 and 100 where 100 is best health. GP access to MRI increased patients' scores on this scale by a net 3.65 points, with a $95 \% \mathrm{Cl}$ of 1.03 to 6.28. This was statistically significant $(P=0.007)$, but again the Cls exclude the target difference of 6.75 points. Furthermore, this difference is much lower than the 16 points judged important by patients responding to a health transition question, ${ }^{35}$ during the development of the KQoL-26. ${ }^{30}$

\section{Implications for future research and clinical practice}

The UK Department of Health has plans to reduce waiting times for diagnostic tests and to upgrade diagnostics within the NHS. ${ }^{17}$ This trial questions whether the small, clinical benefits from GP access to MRI are worth the cost of imaging. The accompanying article on cost-effectiveness analysis ${ }^{21}$ therefore reports the costs and benefits in quality of life of GP access to MRI.

\section{Funding body}

Medical Research Council/Department of Health Primary Care Initiative - Second Call for Proposals (Project Number G0001133). International Standardised Randomised Controlled Trial Number 52135255. The NHS in England, Scotland, and Wales funded the excess treatment and service support costs for the timely provision of MRI and orthopaedic consultation

\section{Ethical approval}

Northern and Yorkshire Multi-Centre Research Ethics Committee approved the study (MREC/1/3/59)

\section{Competing interests}

The authors have stated that there are none

\section{Acknowledgements}

We are indebted to the patients who agreed to take part in this study. The contribution of staff in primary and secondary care to the recruitment of patients and collection of data is greatly appreciated, as is the advice and support 
from the Trial Steering Committee. All members of the DAMASK trial team include Lazaros Andronis, Christine Atwell, Stephen Brealey, Stirling Bryan, Sue Collins, Helen Cox, Ben Cross, Simon Coulton, Fiona Fylan, Andrew Garratt, Fiona Gilbert, Maureen Gillan, Maggie Hendry, Kerenza Hood, Helen Houston, David King, Veronica Morton, Jo Orchard, Michael Robling, lan Russell, David Torgerson, Val Wadsworth, and Clare Wilkinson. A list of clinical collaborators who contributed to designing and delivering presentations at the educational seminars and who supported the trial at their respective NHS sites can be found in the online version of this article. The following is a list of all clinical collaborators who contributed to designing and delivering presentations at the educational seminars and who supported the trial at their respective NHS sites: Dr David Fowler, GP, Kincoth Medical Practice, Aberdeen; Tom Scotland, consultant orthopaedic surgeon, Grampian University Hospitals NHS Trust, Aberdeen; Dr Graeme Houston, consultant radiologist and $\mathrm{Dr}$ Graeme Foubister, consultant orthopaedic surgeon, Ninewells Hospital, Dundee; Dr A Ross, GP, Brechin Medical Practice, Brechin; Dr lan Beggs, consultant radiologist and Dr John Keating, consultant orthopaedic surgeon, Royal Infirmary, Edinburgh; Dr Harry Burnett, GP, Oxgangs Path Surgery, Edinburgh; Dr Nefyn Williams, GP, Llanfairfechan Health Centre, Llanfairfechan; Dr Malcolm Greensmith, consultant radiologist, James Wootton, consultant orthopaedic surgeon, and Aloysius Mbako, orthopaedic surgeon, Wrexham Maelor Hospital NHS Trust, Wrexham; Dr Robert Byrne, consultant radiologist, Dr David Widdowson, consultant radiologist, and Salah Bastawrous, consultant orthopaedic surgeon, Glan Clwyd Hospital NHS Trust, Rhyl; Dr Catrin Barwick, consultant radiologist and Luke McSweeney, consultant orthopaedic surgeon, Ysbytty Gwynedd; Dr John Moroney, GP, Minster Health, York; Anthony Gibbon, consultant orthopaedic surgeon, York NHS Trust; Dr Mark Brook, GP, Wrose Health Centre, Bradford; Dr David Ennion, consultant radiologist and Stephen Bollen, consultant orthopaedic surgeon, Bradford Royal Infirmary Bradford; Dr Julie Kitlowski, GP, St Ann's Medical Centre, Rotherham; Dr Sue Varkey, consultant radiologist and Amanda Rees, consultant orthopaedic surgeon, Rotherham General Hospital, Rotherham; Dr Robert Cooper, consultant radiologis and Jeremy Brown, consultant orthopaedic surgeon, Northern General Hospital, Sheffield; Dr Dan Roper, GP, Springhead Medical Centre, Hull; Dr Damien Taylor, consultant radiologist and Frank Howell, consultant orthopaedic surgeon, Hull Royal Infirmary, Hull.

\section{Discuss this article}

Contribute and read comments about this article on the Discussion Forum: http://www.rcgp.org.uk/bjgp-discuss

\section{REFERENCES}

1. McCormick A, Fleming D, Charlton J. Morbidity statistics from general practice: fourth national study 1991-92. London: HMSO, 1995.

2. Mackenzie R, Dixon AK, Keene GS, et al. Magnetic resonance imaging of the knee: assessment of effectiveness. Clin Radiol 1996; 51(4): 245-250.

3. Mackenzie R, Logan BM, Shah NJ, et al. Direct anatomical-MRI correlation: the knee. Surg Radiol Anat 1994; 16(2): 183-192.

4. Fischer SP, Fox JM, Del Pizzo W, et al. Accuracy of diagnosis from magnetic resonance imaging of the knee: a multi centre analysis of one thousand and fourteen patients. J Bone Joint Surg 1991; 73(1): 2-10.

5. Southgate J, Thomas N. MRI should be used selectively [letter]. BM] 1995; 312(7034): 849.

6. Mackenzie R, Edwards D, Dixon AK. Patients should be seen by an experienced orthopaedic clinician [letter]. BMJ 1996; 312(7034): 849-850.

7. Mackenzie R, Palmer CR, Lomas DJ, Dixon AK. Magnetic resonance imaging of the knee: diagnostic performance studies. Clin Radiol 1996; 51(4): 251-257.

8. Rappeport ED, Mehta S, Weislander SB, et al. MR imaging before arthroscopy in knee joint disorders? Acta Radiol 1996; 37(5): 601.

9. Jackson JL, O’Malley PG, Kroenke K. Evaluation of acute knee pain in primary care. Ann Intern Med 2003; 139(7): 575-588.

10. Watura R, Lloyd DC, Chawda S. Magnetic resonance imaging of the knee: direct access for general practitioners. $B M J 1995 ; 311(7020)$ : 1614.

11. Weinstabl R, Muellner T, Vecsei V, et al. Economic considerations for the diagnosis and therapy of meniscal lesions: can magnetic resonance imaging help reduce the expense? World J Surg 1997; 21(4): 363-368.

12. Chawda SJ, Watura R, Lloyd DC. Magnetic resonance imaging of the lumbar spine: direct access for general practitioners. Br J Gen Pract 1997; 47(422): 575-576.

13. Deyo RA. MRI of the lumbar spine - terrific test or tar baby? New Engl J Med 1994; 331(2): 115-116.

14. Mackenzie R, Dixon AK. Measuring the effects of imaging: an evaluative framework. Clin Radiol 1995; 50(8): 513-518.

15. Robling MR, Houston H, Kinnersley P, et al. An evaluation of general practitioners' use of magnetic resonance imaging. London: NHS Executive, 1998.

16. Department of Health. The NHS improvement plan: putting people at the heart of public services. Norwich: The Stationery Office, 2004

17. Barrett A. Waiting times for scans to decrease, vows Department of Health. BMJ 2005; 331(7511): 256.

18. Department of Health, Hospital Activity Statistics. Imaging and radiodiagnostic data files. http://www.performance.doh.gov.uk/hospital activity/data_requests/imaging_and_radiodiagnostics.htm (accessed 28 Aug 2008).

19. Medical Research Council. A framework for development and evaluation of RCTs for complex interventions to improve health. London: Medical Research Council, 2000.

20. DAMASK Trial Team. Influence of magnetic resonance imaging of the knee on GPs' decisions: a randomised trial. Br J Gen Pract 2007; 57(541): 622-629.

21. DAMASK Trial Team. Cost-effectiveness of magnetic resonance imaging of the knee for patients presenting in primary care. $\mathrm{Br} J \mathrm{Gen}$ Pract 2008; 58(556): 775-778.

22. Brealey SD, Atwell C, Bryan S, et al. The DAMASK trial protocol: a pragmatic randomised trial to evaluate whether GPs should have direct access to MRI for patients with suspected internal derangement of the knee. BMC Health Serv Res 2006; 6: 133.

23. Robling MR, Kinnersley $\mathrm{P}$, Houston $\mathrm{H}$, et al. The impact of guidelines and different dissemination strategies on GPs' knowledge of magnetic resonance imaging. Med Educ 1999; 33(10): 777-779.

24. Eccles M, Steen N, Grimshaw J, et al. Effect of audit and feedback, and reminder messages on primary-care radiology referrals: a randomised trial. Lancet 2001; 357(9266): 1406-1409.

25. Edwards P, Roberts I, Clarke M, et al. Increasing response rates to postal questionnaires: systematic review. BMJ 2002; 324(7347): 1183.

26. Garratt AM, Ruta DA, Abdalla MI, Buckingham JK, Russell IT. The SF36 health survey questionnaire: an outcome measure suitable for routine use within the NHS? BMJ 1993; 306(6890): 1440-1444.

27. EuroQol Group. EuroQol: a new facility for the measurement of health-related quality of life. The EuroQol Group. Health Policy 1990; 16(3): 199-208.

28. Hollingworth W, Mackenzie R, Todd CJ, Dixon AK. Measuring changes in quality of life following magnetic resonance imaging of the knee: SF-36, EuroQol or Rosser index? Qual Life Res 1995; 4(4): 325-334.

29. Garratt AM, Brealey S, Gillespie WJ, DAMASK Trial Team. Patientassessed health instruments for the knee: a structured review. Rheumatology 2004; 43(11): 1414-1423.

30. Garratt AM, Brealey S, Robling M, et al. Development of the Knee Quality of Life (KQoL-26) 26-item questionnaire: data quality, reliability, validity and responsiveness. Health Qual Life Outcomes 2008; 6(1): 48.

31. Brown H, Prescott R. Applied mixed models in medicine. 2nd edn. Chichester: Wiley, 2006.

32. Freedman LS. Evaluating and comparing imaging techniques: a review and classification of study designs. Br J Radiol 1987; 60(719): 1071-1081.

33. Vincken PWL, ter Braak BP, van Erkell AR, et al. Effectiveness of MR imaging in selection of patients for arthroscopy of the knee. Radiology 2002; 223(3): 739-746.

34. Bryan S, Bungay HP, Weatherburn G, Field S. Magnetic resonance imaging for investigation of the knee joint: a clinical and economic evaluation. Int J Technol Assess Health Care 2004; 20(2): 222-229.

35. Guyatt GH, Norman GR, Juniper EF, Griffith LE. A critical look at transition ratings. J Clin Epidemiol 2002; 55(9): 900-908. 